\title{
Efficacy and safety of a balanced salt solution versus a $0.9 \%$ saline infusion for the prevention of contrast-induced acute kidney injury (BASIC trial): a study protocol for a randomized controlled trial
}

Hyung Ah Jo ${ }^{1 \dagger}$, Sehoon Park ${ }^{2 \dagger}$, Chan-Duck Kim³ ${ }^{3}$, Hee-Yeon Jung ${ }^{3}$, Jang-Hee Cho ${ }^{3}$, Ran-hui Cha ${ }^{4}$, Ea Wha Kang ${ }^{5}$, Tae Ik Chang ${ }^{5}$, Sejoong Kim ${ }^{6}$, Hyung-Jong Kim, Byung Ha Chung ${ }^{8}$, Jung Pyo Lee ${ }^{9}$, Jung Tak Park ${ }^{10}$, Seung Hyeok Han ${ }^{10}$, Tae-Hyun Yoo ${ }^{10}$, Dong-Ryeol Ryu ${ }^{11}$, Sung Jin Moon ${ }^{12}$, Jae Hyun Chang ${ }^{13}$, Dong Ki Kim ${ }^{1}$ and Kwon Wook Joo ${ }^{1 *}$

\begin{abstract}
Background: Contrast-induced acute kidney injury (Cl-AKI) is one of the most common causes of iatrogenic kidney injury and, therefore, its prevention is an important issue. However, whether the administration of $0.9 \%$ saline is the optimal prophylaxis method remains uncertain due to its supra-physiologic chloride component. In particular, recent studies suggest that chloride-restricted solutions showed superiority over $0.9 \%$ saline in several clinical settings.

Methods/design: The investigators designed a multicenter randomized controlled trial to compare the efficacy of a balanced salt solution and $0.9 \%$ saline in Cl-AKI prophylaxis. This study will recruit patients who are scheduled for contrast-enhanced computed tomography (CT) scans with CI-AKI prophylaxis. In this study, participants will be randomized into two study arms; the study group will receive a balanced salt solution, and the control group will receive $0.9 \%$ saline. Fluids will be administered as designated in the protocol before and after the CT scan, and an evaluation of baseline clinical status will be performed by obtaining blood and urine samples. During the follow-up visits, the incidence of $\mathrm{Cl}-\mathrm{AKI}$ and long-term outcomes, including the start of renal replacement therapy or all-cause mortality, will be assessed.
\end{abstract}

Discussion: To our knowledge, this study will be the first study assessing the preventive value of a balanced salt solution over $0.9 \%$ saline for Cl-AKI. If the trial shows that the balanced salt solution is as effective for Cl-AKI prophylaxis as $0.9 \%$ saline, the use of the balanced salt solution could be promoted due to the reduced possibility of consequent metabolic acidosis compared to $0.9 \%$ saline.

Trials registration: ClinicalTrials.gov, ID: NCT02799368. Registered on 14 June 2016.

Keywords: Contrast-induced acute kidney injury, Computed tomography, Balanced salt solution

\footnotetext{
* Correspondence: junephro@gmail.com

${ }^{\dagger}$ Equal contributors

'Department of Internal Medicine, Seoul National University College of

Medicine, Seoul, South Korea

Full list of author information is available at the end of the article
} 


\section{Background}

Because iodinated contrast media is widely used in current medicine [1, 2], its well-known side effect, contrast-induced acute kidney injury (CI-AKI), has become one of the most common causes of iatrogenic kidney injury [3, 4]. CI-AKI has been related to increased mortality, longer hospital stays and renal failure in patients with chronic kidney disease (CKD) [3, 5-9]. Hence, preventing CI-AKI has been regarded as an important medical issue $[7,10,11]$, and the use of $0.9 \%$ saline has been an essential part of CI-AKI prophylaxis [10, 12]. However, $0.9 \%$ saline has a $\mathrm{pH}$ of approximately 5.5 and contains a supra-physiologic chloride level. Therefore, the use of saline could cause metabolic acidosis, which contributes to renal vasoconstriction $[13,14]$. From this clinical point of view, a few trials have tested the efficacy of a sodium bicarbonate fluid solution for CI-AKI prophylaxis, but the results failed to show consistent superiority over $0.9 \%$ saline [15]. From another aspect, a recent clinical trial compared the use of $0.9 \%$ saline and no prophylaxis for patients with reduced kidney function, and showed no certain benefits with $0.9 \%$ saline prophylaxis. However, the effect of CI-AKI prophylaxis may be necessary to be tested in higher-risk patients, such as patients with other risk factors or even lower baseline estimated glomerular filtration rate (eGFR) [16].

Recently, several human studies reported that metabolic acidosis and vasoconstriction are less pronounced when using a balanced salt solution, which has a physiologic level of chloride and a neutral $\mathrm{pH}$, compared to using $0.9 \%$ saline $[17,18]$. Additionally, there were prospective studies suggesting that using chloriderestrictive solutions, rather than using chloride-rich solutions, for fluid resuscitation can reduce acute kidney injury (AKI) in critically ill patients $[19,20]$. In accordance with these findings, a large-scale cohort study was reported, demonstrating the preventive effect of a balanced salt solution for AKI over $0.9 \%$ saline [21]. Additionally, one clinical trial is currently recruiting participants to prove the benefit of chloride-restrictive fluids in cardiac surgery [22]. However, to the investigators' knowledge, there are no ongoing trials regarding the effectiveness of a balanced salt solution for CI-AKI prophylaxis.

This multicenter randomized controlled trial is designed to verify the effectiveness of a balanced salt solution for CI-AKI prophylaxis. After randomization, the study group will receive the balanced salt solution, and the control group will receive $0.9 \%$ saline. In this trial, the investigators will address whether the balanced salt solution could be a potential standard solution for CI-AKI prophylaxis.

\section{Methods/design}

The study is a randomized, open-label, active-control, two-parallel-group, multicenter, phase-III study. The study protocol is summarized in Figs. 1 and 2, the latter showing the study timeline which accords with the Standard Protocol Items: Recommendations for Interventional Trials (SPIRIT) Figure. The main goal is to evaluate whether using a balanced salt solution

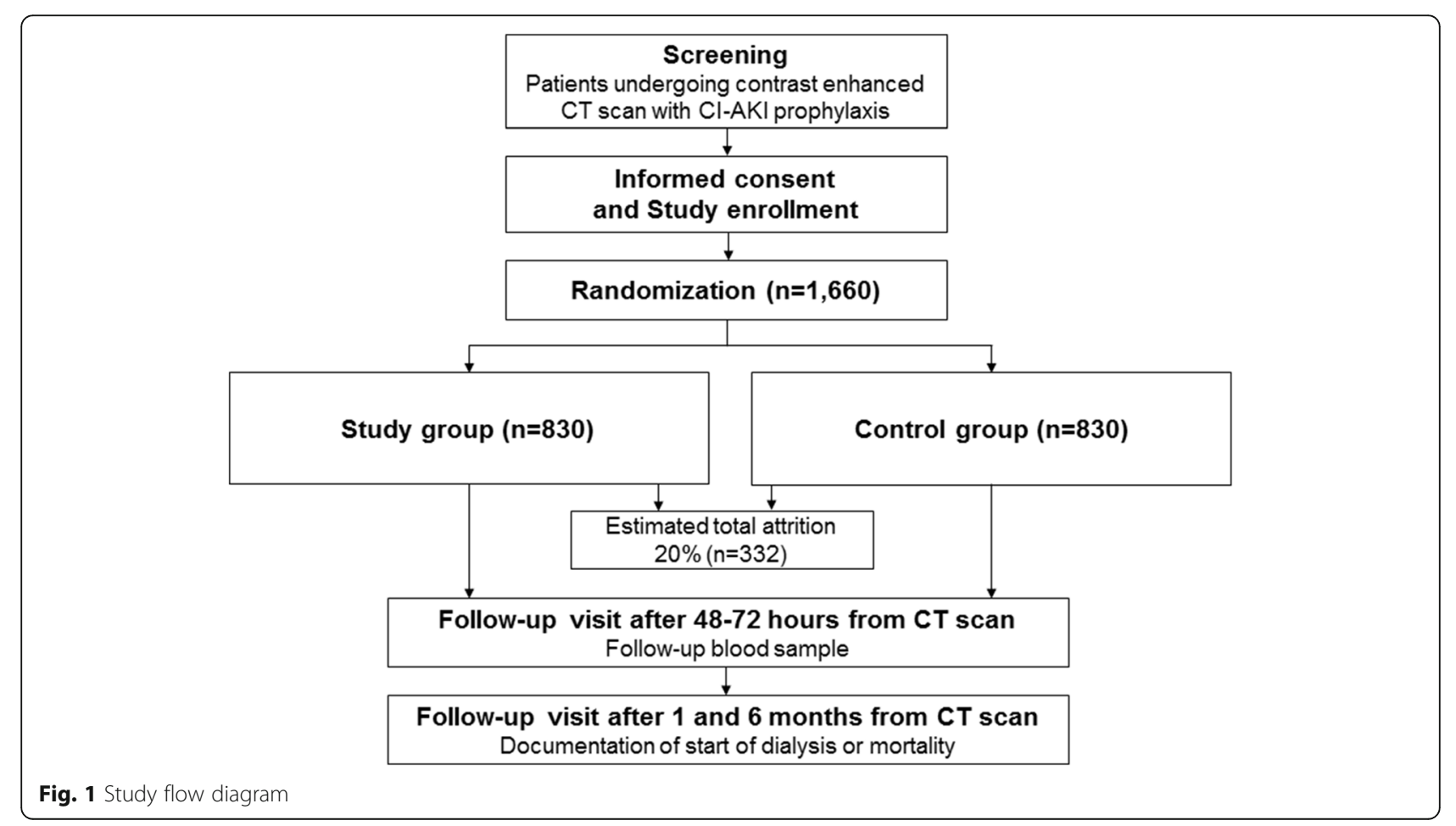




\begin{tabular}{|c|c|c|c|c|c|c|}
\hline & $\begin{array}{c}\text { Screening } \\
\text { and } \\
\text { randomizati } \\
\text { on }\end{array}$ & Baseline & $\begin{array}{l}\text { Treatment } \\
\text { period }\end{array}$ & $\begin{array}{c}\text { Assessment } \\
1\end{array}$ & $\begin{array}{c}\text { Assessment } \\
2^{*}\end{array}$ & $\begin{array}{c}\text { Assessment } \\
3^{*}\end{array}$ \\
\hline Visit & \multicolumn{3}{|c|}{1} & 2 & 3 & 4 \\
\hline Week & $<-3 h$ & $-1 h$ & $-1 \mathrm{~h} \sim 4 \mathrm{~h}$ & $48 \mathrm{~h}-72 \mathrm{~h}$ & $1 \mathrm{~m}$ & $6 \mathrm{~m}$ \\
\hline Informed consent & $\mathrm{O}$ & & & & & \\
\hline Inclusion/exclusion criteria & $\mathrm{O}$ & & & & & \\
\hline Demographic data & $\mathrm{O}$ & & & & & \\
\hline Concomitant medications & $\mathrm{O}$ & & & $\mathrm{O}$ & $\mathrm{O}$ & $\mathrm{O}$ \\
\hline Physical examination & $\mathrm{O}$ & & & $\mathrm{O}$ & $\mathrm{O}$ & $\mathrm{O}$ \\
\hline Vital sign & $\mathrm{O}$ & $\mathrm{O}^{* *}$ & $\mathrm{O}$ & $\mathrm{O}$ & & \\
\hline randomization & $\mathrm{O}$ & & & & & \\
\hline $\begin{array}{c}\text { Body weight, height, body } \\
\text { mass index }\end{array}$ & $\mathrm{O}$ & & & & & \\
\hline Hemoglobin & & $\mathrm{O}$ & & & & \\
\hline Serum Chemistry ${ }^{1}$ & & $\mathrm{O}$ & & $\mathrm{O}^{3}$ & & \\
\hline Urine chemistry $^{2}$ & & $\mathrm{O}^{* *}$ & & & & \\
\hline $\begin{array}{l}\text { Estimated glomerular } \\
\text { filtration rate }\end{array}$ & & $\mathrm{O}$ & & $\mathrm{O}$ & & \\
\hline Study fluid administration & & & $\mathrm{O}$ & & & \\
\hline $\begin{array}{c}\text { Documentation of mortality } \\
\text { or dialysis }\end{array}$ & & & & & $\mathrm{O}$ & $\mathrm{O}$ \\
\hline Adverse event monitoring & & & Contin & huously & & \\
\hline $\begin{array}{l}{ }^{1} \text { Serum chemistry = Protein, } \\
\mathrm{CO}_{2} \\
{ }^{2} \text { Urine chemistry = Spot urine } \\
{ }^{3} 48-72 \mathrm{~h} \text { laboratory test: Creati } \\
{ }^{*} \text { Telephone interview will be } \\
\text { circumstances to visit hospital } \\
{ }^{* *} \text { If vital sign or urine chemist } \\
\text { from screening period. }\end{array}$ & $\begin{array}{l}\text { Albumin, Blo } \\
\text { total protein } \\
\text { inine, Sodium } \\
\text { carried out } \\
\text { try informatio }\end{array}$ & $\begin{array}{l}\text { d urea nitr } \\
\text { reatinine } \\
\text { Potassium } \\
\text { ased on in } \\
\text { is not avai }\end{array}$ & $\begin{array}{l}\text { Chloride, To } \\
\text { rmed conse } \\
\text { able, the info }\end{array}$ & $\begin{array}{l}\mathrm{tal} \mathrm{CO}_{2} \\
\text { nt when parti }\end{array}$ & $\begin{array}{l}\text { icipants are } \\
\text { d be substitute }\end{array}$ & $\begin{array}{l}\text { Chloride, Tota } \\
\text { inder difficul } \\
\text { ed with results }\end{array}$ \\
\hline
\end{tabular}

Fig. 2 Study timeline and endpoints

is non-inferior to the use of $0.9 \%$ saline for CI-AKI prophylaxis. In current medicine, many clinics provide CI-AKI prophylaxis for patients with decreased kidney function, and this study will recruit these patients who are scheduled for contrast-enhanced computed tomography (CT) scans. After participants are enrolled in the study with informed consent, they will be randomized into the two study arms; the study group will receive a balanced salt solution, and the control group will receive $0.9 \%$ saline. The fluids for CI-AKI prophylaxis will be administered as designated in the protocol in the daycare center before and after the CT scan. The baseline clinical status will be evaluated by obtaining blood and urine samples. Next, the participants will be scheduled for a follow-up visit, and their incidence of CI-AKI and further prognosis will be evaluated. The protocol has been designed according to the SPIRIT guidelines (see Additional file 1.)

\section{Study participants}

The study will recruit participants from the following 12 tertiary hospitals in Korea: Seoul National University Hospital, Kyungpook National University Hospital, National Medical Center, National Health Insurance Service Ilsan Hospital, Seoul National University Bundang Hospital, Bundang CHA Medical Center, Seoul St. Mary's Hospital, Seoul National University Boramae Medical Center, Severance Hospital, Ewha Womans University Mokdong 
Hospital, Catholic Kwandong University International St. Mary's Hospital, and Gachon University Gil Medical Center. The planned duration for the study enrollment completion is 2 years. Below, we describe the inclusion and exclusion criteria for the study participants, and the age or eGFR cutoff values are based on a current clinical guideline [11].

Participants' inclusion criteria are as follows:

1. Adult patients (age 18 years or older) who undergo iodinated contrast-enhanced CT scans

2. Patients with a baseline eGFR, which is measured within 3 months, of less than $45 \mathrm{~mL} / \mathrm{min} / 1.73 \mathrm{~m}^{2}$ or both having a baseline eGFR less than $60 \mathrm{~mL} / \mathrm{min} /$ $1.73 \mathrm{~m}^{2}$ and either one of the following risk factors:

(a) diabetes mellitus, or

(b)age 60 years or older

3. Patients who are able to provide informed consent and give adequate information for the endpoint assessment

Participants' exclusion criteria are as follow:

1. Patients with a baseline eGFR of less than $15 \mathrm{~mL} /$ $\mathrm{min} / 1.73 \mathrm{~m}^{2}$ or who are on dialysis

2. Heart failure with a left ventricular ejection fraction $<45 \%$ or severe symptoms (New York Heart Association functional classification III or IV)

3. Co-existing acute pulmonary edema or decompensated heart failure requiring the following medications: dobutamine, dopamine, milrinone, amrinone, or nesiritide

4. Patients with last measured serum potassium level > $5.5 \mathrm{mEq} / \mathrm{L}$ or serum sodium level $>145 \mathrm{mEq} / \mathrm{L}$ at the screening period or within 3 months before the CT scan

5. History of intravenous or intra-arterial administration of contrast agent within 1 week

6. Previous history of hypersensitivity reaction to the iodinated contrast agent

7. History of multiple myeloma

8. Women who are currently pregnant/breastfeeding or planning pregnancy

9. Patients with an expected survival duration of less than 6 months

10.Patients who are already enrolled in another clinical trial

\section{Intervention protocols and fluids}

The main intervention is the intravenous administration of fluids for CI-AKI prophylaxis. The two types of fluid used in our study are plasma solution A and $0.9 \%$ saline. The study fluid, plasma solution A, will be manufactured by CJ HealthCare Corporation, Seoul, South Korea and in facilities following standards of Good Manufacturing
Practice. The provided product volume will be $1000 \mathrm{~mL}$, and plasma solution A will contain $5.26 \mathrm{~g}$ of sodium chloride, $5.02 \mathrm{~g}$ of sodium gluconate, $3.68 \mathrm{~g}$ of sodium acetate hydrate, $0.37 \mathrm{~g}$ of potassium chloride and $0.3 \mathrm{~g}$ of magnesium chloride. In contrast, $0.9 \%$ saline will have $9.0 \mathrm{~g}$ of sodium chloride with a volume of $1000 \mathrm{~mL}$. The concentrations of each component of the two fluids are summarized in Table 1. Plasma solution A, with $98 \mathrm{mEq} / \mathrm{L}$ of chloride, is the balanced salt solution used in this study and will be administered to the study group. Meanwhile, $0.9 \%$ saline, which is the standard fluid for CI-AKI prophylaxis and contains $154 \mathrm{mEq} / \mathrm{L}$ of chloride, will be used in the control group for CI-AKI prophylaxis. Both fluids will be administered by the designated rate, i.e., $3 \mathrm{~mL} / \mathrm{kg} / \mathrm{h}$ for $1 \mathrm{~h}$ before and $1.5 \mathrm{~mL} / \mathrm{kg} / \mathrm{h}$ for $4 \mathrm{~h}$ after the CT scans. Participants will remain fasting during the fluid administration. All CT scans will use iso- or low-osmolar contrast agents. $N$ acetylcysteine will not be administered to the participants in this study as this medication lacks a definite benefit for CI-AKI prophylaxis [23]. Other medication use will not be restricted in the study.

\section{Study endpoint}

The primary endpoint of the study is the incidence of CI-AKI. The event of CI-AKI will be defined as an increase in the serum creatinine level $\geq 0.5 \mathrm{mg} / \mathrm{dl}$ or $\geq 25 \%$ from baseline at 48-72 $\mathrm{h}$ after the CT scan [24]. For the assessment of the primary endpoint, the patients will visit the study hospital and a follow-up blood sample will be drawn; along with other tests, serum creatinine will be measured by the method that has been standardized to isotope dilution mass spectrometry. Next, two secondary endpoints will be evaluated in the study; one is the eGFR decrement at 48-72 $\mathrm{h}$ after the CT scan, and the other is the start of dialysis, or mortality, which will be assessed at 1 and 6 months, respectively. The eGFR decrement will be assessed by the baseline and follow-up eGFR values calculated by the serum creatinine as measured in the primary endpoint assessment. The eGFR will

Table 1 Components of the two fluids used in the study

\begin{tabular}{lll}
\hline & $0.9 \%$ saline & Plasma solution A \\
\hline $\mathrm{Na}^{+}(\mathrm{mEq} / \mathrm{L})$ & 154 & 140 \\
$\mathrm{~K}^{+}(\mathrm{mEq} / \mathrm{L})$ & & 5 \\
$\mathrm{Ca}^{2+}(\mathrm{mEq} / \mathrm{L})$ & & \\
$\mathrm{Mg}^{2+}(\mathrm{mEq} / \mathrm{L})$ & & 1.5 \\
$\mathrm{Cl}^{-}(\mathrm{mEq} / \mathrm{L})$ & 154 & 98 \\
Acetate $(\mathrm{mEq} / \mathrm{L})$ & & 27 \\
Gluconate $(\mathrm{mEq} / \mathrm{L})$ & & 23 \\
Osmolarity $(\mathrm{mOsm} / \mathrm{L})$ & 308 & 295 \\
$\mathrm{pH}$ & 6.0 & 7.4 \\
\hline
\end{tabular}


be calculated using the Modification of Diet in Renal Disease (MDRD) equation [25]. For documentation of mortality and the beginning of dialysis, the study participants will be questioned via a direct visit or phone poll.

\section{Sample size}

The incidence of CI-AKI when using $0.9 \%$ saline for prophylaxis was predicted according to the previous prospective study (11.5\%) [26]. In contrast, there was no study regarding the incidence of CI-AKI after the use of a balanced salt solution; therefore, the expected incidence of CI-AKI in the study group was derived from the prospective study, which compared the incidence of AKI in an intensive care unit population according to the resuscitation fluid use (8.4\%) [20]. With a noninferiority limit of $1.5 \%$, a total of 1660 study participants (830 in each group) would result in a power of at least $80 \%$ with a one-sided type- 1 error rate $(\alpha)$ of $2.5 \%$, allowing a $20 \%$ withdrawal rate in each group.

\section{Blinding and randomization}

The study is an open-label study; therefore, the type of administered fluid will be disclosed both to the investigators and the participants. The participants and their information will be assigned a unique identifier number at the time of initial enrollment and stored in a webbased data collection system. The group allocation will be performed after randomization in a 1:1 manner, at least $3 \mathrm{~h}$ before contrast material administration. The randomization scheme will be generated by using the on-line randomization service developed by Sealed Envelope Ltd. (www.sealedenvelope.com).

\section{Statistical analysis}

All primary and secondary endpoints and serious adverse effects will be analyzed by the investigators at the participating hospitals. The final analysis will be completed at 6 months after the last participant's trial. The primary endpoint, i.e., the incidence of CI-AKI in the study and control groups will be compared by a non-inferiority test with $1.5 \%$ as the non-inferiority margin. The eGFR decrement, one of the secondary endpoints in our study, will be evaluated by chi-squared test. End-stage renal disease (ESRD) progression and mortality will be assessed by Kaplan-Meier survival curve with a log-rank test.

\section{Data management}

All participants' information related to the study will be recorded in the Case Reporting Format (CRF) and recorded in an electronic, password-protected database. Study participants will only be recognized by their study ID, and their personal identifier will not be recorded and stored. All records will be accessed by the investigators and authorized personnel only to secure confidentiality.
The investigators at each participating hospital will monitor the completeness of the CRF. The database will be locked and maintained for 10 years only for the purpose of a secondary analysis or investigations by the attending Institutional Review Boards (IRBs) and the Korean Ministry of Food and Drug Safety (MFDS).

\section{Adverse events}

During the entire study period and after 30 days from the end of the trial, adverse events $(\mathrm{AE})$ will be reported and recorded in the participants' CRF. The severity of AEs will be graded as mild, moderate and severe, and their relationship between the study groups will also be assessed by clinical judgement. If an $\mathrm{AE}$ requiring hospitalization or causing a medically critical situation occurs, the event will be recorded as a severe adverse event (SAE). All SAEs will be reported to the investigator of the attending hospital and the IRB of reference within $24 \mathrm{~h}$ after the information has been collected. AEs that cannot deny a relationship to the study must be followed until the AEs have been resolved. Whenever AEs progress to the level of SAEs, the events must also be reported according to the above protocol. At the time of the study result submission, AEs and their relationship to the study will be documented in a table and submitted.

\section{Discussion}

Prophylaxis for CI-AKI is an important clinical issue because $\mathrm{CI}-\mathrm{AKI}$ is common and worsens patient prognosis. Although the use of $0.9 \%$ saline has been the standard method for CI-AKI prophylaxis, its potentially harmful effect was addressed in previous studies. There were several efforts to find a more appropriate infusion fluid for CI-AKI prophylaxis, but trials with sodium bicarbonate fluid failed to demonstrate prophylactic efficacy. The investigators considered the balanced salt solution to be a potential fluid, which can replace $0.9 \%$ saline, due to the balanced salt solution's physiologic components and evidence from recent study results. To our knowledge, the study will be the first study assessing the preventive value of a balanced salt solution over $0.9 \%$ saline for CI-AKI. If the proposed trial demonstrates that the balanced salt solution is effective for CI-AKI prophylaxis, the use of the fluid could be promoted due to its reduced likelihood of inducing metabolic acidosis. Therefore, the results of this trial could be useful to improve the prophylaxis method and, consequently, decrease the incidence of CI-AKI and its adverse outcomes.

\section{Trial status}

The BASIC randomized controlled clinical study has received governance approval and is registered at 
ClinicalTrials.gov (NCT02799368). The trial started recruitment in November, 2016.

\section{Additional file}

Additional file 1: SPIRIT Checklist for the present study protocol. (DOC $113 \mathrm{~kb}$ )

\section{Abbreviations}

AE: Adverse event; AKI: Acute kidney injury; Cl-AKI: Contrast-induced acute kidney injury; CKD: Chronic kidney disease; CRF: Case Reporting Format; $\mathrm{CT}$ : Computed tomography; eGFR: Estimated glomerular filtration rate; IRB: Institutional Review Board; MFDS: Ministry of Food and Drug Safety; SAE: Severe adverse event

\section{Acknowledgements}

None

\section{Funding}

This study is funded by CJ HealthCare Corporation (CS2015_0046), Seoul, Korea.

\section{Availability of data and materials}

The final trial dataset will only be accessible to the study investigators.

\section{Authors' contributions}

KWJ is the principal investigator and supervised the overall project. DKK gave the research idea and helped in the manuscript correction, provided statistical knowledge and recruiting study participants from Seoul National University Hospital. SP participated in the study design and wrote the original manuscript. HAJ assisted in the study design, revised the manuscript, reviewed the ethical aspects of the study and acquired IRB/government approval. CDK is leading the study at Kyunpook University Hospital and reviewed the study protocol. HYJ helped in study design and participating in patient recruitment. JHC helped in revising the study protocol and participating in patient recruitment. $\mathrm{RHC}$ is leading the study in the National Medical Center and helped in statistical design. EWK provided statistical knowledge for revising the study protocol and participated in patient recruitment. TIC is leading the study in the National Health Insurance Service Ilsan Hospital. SJK is leading the study at Seoul National University Bundang Hospital and provided background knowledge of the study. HJK is leading the study at Bundang CHA hospital and participated in the study design. $\mathrm{BHC}$ is leading the study at St. Mary's hospital and contributed to the study concept. JPL is leading the study at Seoul National University Boramae Hospital. JTP is recruiting study participants at Severance Hospital and helped in correcting the study protocol. SHH participated in study design and provided study concepts. THY is leading the study at Severance Hospital and helped in writing the study protocol. DRR is leading the study at Ewha Womans University Mokdong Hospital and participated in revising the study protocol. SJM participated in the study initiation at Incheon St. Mary's Hospital. JHC is leading the study at Gachon University Gil Medical Center. All authors read and approved the final manuscript.

\section{Ethics approval and consent to participate}

The study will be performed according to the following common guidelines for clinical trial: the principles of the Declaration of Helsinki - Ethical Principles for Medical Research Involving Human Subjects in 2013 and Good Clinical Practice Guidelines in 2015. The study protocol has been designed and will report results according to current Consolidated Standards of Reporting Trials (CONSORT) guidelines and is registered at ClinicalTrials.gov (NCT02799368). The protocol design has been approved by the Institutional Review Board (IRB) of Seoul National University Hospital (H-1605-156-768) and the Ministry of Food and Drug Safety of Korea (MFDS). All study participants will be informed about the purpose, intervention and risk/ benefit of the study, and only those who provide written consent will be registered to the study.

Kyungpook National University Hospital: KNUH 2016-09-007

National Medical Center: $\mathrm{H}-1608-069-004$

National Health Insurance Service IIsan Hospital: NHIMC 2016-10-010-002
Seoul National University Bundang Hospital: B-1609/363-401 Bundang CHA Hospital: 2016-09-011-002

Seoul St. Mary's Hospital: KC16MIMT0741

Seoul National University Boramae Hospital: 16-2016-126

Severance Hospital: 4-2016-0691

Ewha Womans University Mokdong Hospital: 2016-10-014

Incheon St. Mary's Hospital: IS16MIMT0033

Gachon University Gil Medical Center: GAIRB2016-322

\section{Consent for publication}

Not applicable.

Competing interests

The authors declare that they have no competing interests.

\section{Publisher's Note}

Springer Nature remains neutral with regard to jurisdictional claims in published maps and institutional affiliations.

\section{Author details}

${ }^{1}$ Department of Internal Medicine, Seoul National University College of Medicine, Seoul, South Korea. ${ }^{2}$ Department of Biomedical Sciences, Seoul National University College of Medicine, Seoul, South Korea. ${ }^{3}$ Department of Internal Medicine, Kyungpook National University Hospital, Daegu, South Korea. ${ }^{4}$ Department of Internal Medicine, National Medical Center, Seoul, South Korea. ${ }^{5}$ Department of Internal Medicine, National Health Insurance Service IIsan Hospital, Goyang, South Korea. ${ }^{6}$ Department of Internal Medicine, Seoul National University Bundang Hospital, Gyeonggi-do, South Korea. ${ }^{7}$ Department of Internal Medicine, Bundang CHA Medical Center, CHA University, Gyeonggi-do, South Korea. ${ }^{8}$ Department of Internal Medicine, Seoul St. Mary's Hospital, The Catholic University of Korea, Seoul, South Korea. ${ }^{9}$ Department of Internal Medicine, Seoul National University Boramae Medical Center, Seoul, South Korea. ${ }^{10}$ Department of Internal Medicine, Yonsei University College of Medicine, Seoul, South Korea. ${ }^{11}$ Department of Internal Medicine, Ewha Womans University Mokdong Hospital, Seoul, South Korea. ${ }^{12}$ Department of Internal Medicine, Catholic Kwandong University International St. Mary's Hospital, Incheon, South Korea. ${ }^{13}$ Department of Internal Medicine, Gachon University Gil Medical Center, Incheon, South Korea.

Received: 18 November 2016 Accepted: 13 September 2017

Published online: 05 October 2017

\section{References}

1. Go AS, et al. Heart disease and stroke statistics - 2013 update: a report from the American Heart Association. Circulation. 2013:127(1):e6-e245.

2. Ludwig U, Keller F. Prophylaxis of contrast-induced nephrotoxicity. Biomed Res Int. 2014;2014:308316.

3. Rihal CS, et al. Incidence and prognostic importance of acute renal failure after percutaneous coronary intervention. Circulation. 2002;105(19):2259-64.

4. Nash K, Hafeez A, Hou S. Hospital-acquired renal insufficiency. Am J Kidney Dis. 2002;39(5):930-6.

5. McCullough PA, et al. Acute renal failure after coronary intervention: incidence, risk factors, and relationship to mortality. Am J Med. 1997;103(5):368-75.

6. Weisbord SD, et al. Incidence and outcomes of contrast-induced AKI following computed tomography. Clin J Am Soc Nephrol. 2008;3(5):1274-81.

7. James MT, et al. Acute kidney injury following coronary angiography is associated with a long-term decline in kidney function. Kidney Int. 2010; 78(8):803-9.

8. Park $\mathrm{S}$, et al. Contrast-induced nephropathy after computed tomography in stable CKD patients with proper prophylaxis: 8-year experience of outpatient prophylaxis program. Medicine (Baltimore). 2016;95(18):e3560.

9. Kim SM, et al. Incidence and outcomes of contrast-induced nephropathy after computed tomography in patients with CKD: a quality improvement report. Am J Kidney Dis. 2010;55(6):1018-25.

10. Barrett BJ, Parfrey PS. Preventing nephropathy induced by contrast medium. N Engl J Med. 2006;354(4):379-86.

11. Ellis $\mathrm{JH}$, et al. ACR manual on contrast media. ACR Committee on Drugs and Contrast Media (Version 10.3). American College of Radiology website. http://wwwacr.org/Quality-Safety/Resources/Contrast-Manual. 2017 Accessed 7 Aug 2017. 
12. Mueller C. Prevention of contrast-induced nephropathy with volume supplementation. Kidney Int Suppl. 2006;100:S16-19.

13. Yunos NM, et al. Bench-to-bedside review: chloride in critical illness. Crit Care. 2010;14(4):226.

14. Wilcox CS. Regulation of renal blood flow by plasma chloride. J Clin Invest. 1983;71(3):726-35.

15. Zoungas $\mathrm{S}$, et al. Systematic review: sodium bicarbonate treatment regimens for the prevention of contrast-induced nephropathy. Ann Intern Med. 2009;151(9):631-8.

16. Nijssen EC, et al. Prophylactic hydration to protect renal function from intravascular iodinated contrast material in patients at high risk of contrastinduced nephropathy (AMACING): a prospective, randomised, phase 3, controlled, open-label, non-inferiority trial. Lancet. 2017;389(10076):1312-22.

17. Chowdhury AH, et al. A randomized, controlled, double-blind crossover study on the effects of 1-L infusions of $6 \%$ hydroxyethyl starch suspended in $0.9 \%$ saline (Voluven) and a balanced solution (Plasma Volume Redibag) on blood volume, renal blood flow velocity, and renal cortical tissue perfusion in healthy volunteers. Ann Surg. 2014;259(5):881-7.

18. Chowdhury AH, et al. A randomized, controlled, double-blind crossover study on the effects of 2-L infusions of $0.9 \%$ saline and plasma-lyte(R) 148 on renal blood flow velocity and renal cortical tissue perfusion in healthy volunteers. Ann Surg. 2012;256(1):18-24.

19. Yunos NM, et al. Chloride-liberal vs. chloride-restrictive intravenous fluid administration and acute kidney injury: an extended analysis. Intensive Care Med. 2015;41(2):257-64.

20. Yunos NM, et al. Association between a chloride-liberal vs chloriderestrictive intravenous fluid administration strategy and kidney injury in critically ill adults. JAMA. 2012;308(15):1566-72.

21. Shaw AD, et al. Impact of intravenous fluid composition on outcomes in patients with systemic inflammatory response syndrome. Crit Care. 2015;19:334.

22. ClinicalTrials.gov [Internet]. Bethesda (MD): National Library of Medicine (US). Identifier NCT02020538, Limiting IV Chloride to Reduce AKI After cardiac surgery (LICRA). 2013. [cited 2017 Sep 7]. Available from: https://clinicaltrials. gov/ct2/show/NCT02020538.

23. Birck R, et al. Acetylcysteine for prevention of contrast nephropathy: metaanalysis. Lancet. 2003;362(9384):598-603.

24. KIDGO. Clinical practice guideline for acute kidney injury. Kidney Int Suppl. 2012;2:116-21

25. Levey AS, et al. National Kidney Foundation practice quidelines for chronic kidney disease: evaluation, classification, and stratification. Ann Intern Med. 2003;139(2):137-47.

26. Brar SS, et al. Sodium bicarbonate vs sodium chloride for the prevention of contrast medium-induced nephropathy in patients undergoing coronary angiography: a randomized trial. JAMA. 2008:300(9):1038-46.

\section{Submit your next manuscript to BioMed Central and we will help you at every step:}

- We accept pre-submission inquiries

- Our selector tool helps you to find the most relevant journal

- We provide round the clock customer support

- Convenient online submission

- Thorough peer review

- Inclusion in PubMed and all major indexing services

- Maximum visibility for your research

Submit your manuscript at www.biomedcentral.com/submit

CBiomed Central 